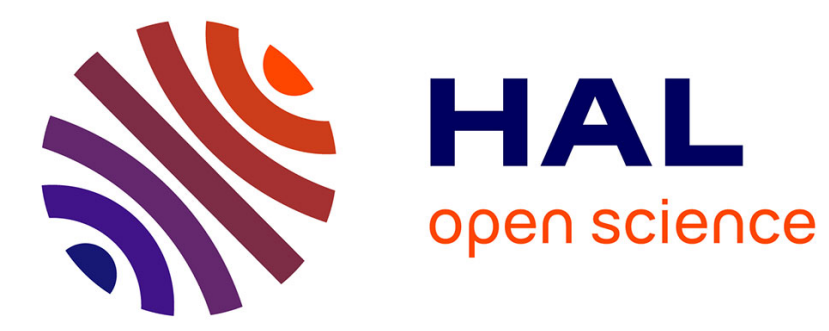

\title{
Control of near-grazing dynamics in impact oscillators
} Harry Dankowicz, Jenny Jerrelind

\section{To cite this version:}

Harry Dankowicz, Jenny Jerrelind. Control of near-grazing dynamics in impact oscillators. Proceedings of the Royal Society A: Mathematical, Physical and Engineering Sciences, 2005, 461 (2063), pp.3365-3380. 10.1098/rspa.2005.1516 . hal-01544507

\section{HAL Id: hal-01544507 \\ https://hal.science/hal-01544507}

Submitted on 8 Jul 2017

HAL is a multi-disciplinary open access archive for the deposit and dissemination of scientific research documents, whether they are published or not. The documents may come from teaching and research institutions in France or abroad, or from public or private research centers.
L'archive ouverte pluridisciplinaire HAL, est destinée au dépôt et à la diffusion de documents scientifiques de niveau recherche, publiés ou non, émanant des établissements d'enseignement et de recherche français ou étrangers, des laboratoires publics ou privés.

\section{다(1)(2)}

Distributed under a Creative Commons Attribution - ShareAlike| 4.0 International 


\title{
Control of near-grazing dynamics in impact oscillators
}

\author{
By Harry Dankowicz ${ }^{1}$ and Jenny Jerrelind ${ }^{2}$ \\ ${ }^{1}$ Department of Engineering Science and Mechanics, MC 0219, Virginia \\ Polytechnic Institute and State University, Blacksburg, VA 24061, USA \\ (danko@vt.edu) \\ ${ }^{2}$ Department of Aeronautical and Vehicle Engineering, \\ Royal Institute of Technology, 10044 Stockholm, Sweden
}

A method is presented for controlling the persistence of a local attractor near a grazing periodic trajectory in a piecewise smooth dynamical system in the presence of discontinuous jumps in the state associated with intersections with system discontinuities. In particular, it is shown that a discrete, linear feedback strategy may be employed to retain the existence of an attractor near the grazing trajectory, such that the deviation of the attractor from the grazing trajectory goes to zero as the system parameters approach those corresponding to grazing contact. The implementation relies on a local analysis of the near-grazing dynamics using the concept of discontinuity mappings. Numerical results are presented for a linear and a nonlinear oscillator.

Keywords: piecewise smooth systems; grazing trajectories; grazing bifurcations; discontinuity mappings; control

\section{Introduction}

The onset of impacting motions of mechanical systems under variations in a system parameter $\mu$ is known to be associated with dramatic and abrupt changes in system response (Nordmark 1991, 1997; Fredriksson \& Nordmark 1997; Dankowicz \& Nordmark 1999; Molenaar et al. 2001; Dankowicz et al. 2002; Dankowicz \& Zhao 2005). Specifically, bifurcations in the long-term system response occur as $\mu$ increases past some critical value $\mu_{\text {grazing, }}$, at which value there exists a periodic nonimpacting oscillation of the mechanical system that achieves zero-relative-velocity contact with an internal or external mechanical limit. In a state-space description of the dynamics of the system, such zero-relative-velocity contact corresponds to a grazing contact between a state-space trajectory and a discontinuity surface, for example, representing the sudden changes in the velocities of the system components that result from an impact. In contrast to periodic trajectories in smooth systems, the local description in the vicinity of a grazing trajectory is well known to be non-differentiable with dramatic implications to the stability of the grazing trajectory and to its persistence under further parameter variations. Indeed, as there is no advance warning of this instability, any local description must account for the non-smooth character of the flow near the grazing trajectory. 
Analysis of the local dynamics in the vicinity of a grazing trajectory for $\mu \approx \mu_{\text {grazing }}$ is made possible through the introduction of a discontinuity mapping that (i) captures the local dynamics in the vicinity of the grazing contact including variations in time-of-flight to the discontinuity and the impact mapping; (ii) can be entirely characterized by conditions at the grazing contact; (iii) is non-smooth in the deviation from the point of grazing contact and (iv) can be studied to arbitrary order of accuracy (Nordmark 1991; Dankowicz \& Nordmark 1999; di Bernardo et al. 2000; Dankowicz \& Zhao 2005). Properly formulated, the discontinuity mapping thus introduces the correction to the otherwise smooth dynamics that is due to the brief interaction with the discontinuity.

As shown by Fredriksson \& Nordmark (1997; but see also Nordmark 1991, 1997), necessary and sufficient conditions for the persistence of a local attractor near the grazing periodic trajectory for $\mu \approx \mu_{\text {grazing }}$ may be formulated in terms of a condition on the linearization of the mechanical system about the grazing trajectory in the absence of impacts. In this paper, we rely on this insight for the formulation of a discrete, closed-loop control strategy that controls the persistence of a local attractor by appropriate real-time changes to the position of the mechanical limit. In particular, we illustrate the possibility of dramatically changing the bifurcation scenario associated with grazing contact with a minimum of control.

The paper is organized as follows. Section 2 presents an analysis of the neargrazing dynamics of a piecewise smooth formulation of a linear, forced impact oscillator and the formulation of a feedback strategy for controlling the persistence of a local attractor near the grazing periodic trajectory. Section 3 illustrates the generality of the analysis and control methodology by considering an application to a nonlinear oscillator. A concluding discussion is presented in $§ 4$.

\section{A linear oscillator}

\section{(a) Mathematical model}

Consider the response of a linear oscillator to a periodic excitation in the presence of perfectly elastic impacts of the oscillator with a mechanical limit at some critical displacement. In particular, suppose that the evolution of the oscillator is governed by the second-order differential equation

$$
\frac{\mathrm{d}^{2} q}{\mathrm{~d} t^{2}}(t)+\frac{2 \gamma}{\omega} \frac{\mathrm{d} q}{\mathrm{~d} t}(t)+\frac{1}{\omega^{2}} q(t)=A\left[\left(\frac{1}{\omega^{2}}-1\right) \cos t-\frac{2 \gamma}{\omega} \sin t\right],
$$

for $q \geq q_{\mathrm{c}}, \omega, \gamma, A>0$, and such that if

$$
q\left(\lim _{t \rightarrow t_{*}^{-}} t\right)=q_{\mathrm{c}} \text { and } \frac{\mathrm{d} q}{\mathrm{~d} t}\left(\lim _{t \rightarrow t_{*}^{-}} t\right) \leq 0,
$$

for some time $t_{*}$, then

$$
q\left(\lim _{t \rightarrow t_{*}^{+}} t\right)=q_{\mathrm{c}} \quad \text { and } \quad \frac{\mathrm{d} q}{\mathrm{~d} t}\left(\lim _{t \rightarrow t_{*}^{+}} t\right)=-\frac{\mathrm{d} q}{\mathrm{~d} t}\left(\lim _{t \rightarrow t_{*}^{-}} t\right) .
$$


In terms of the state vector

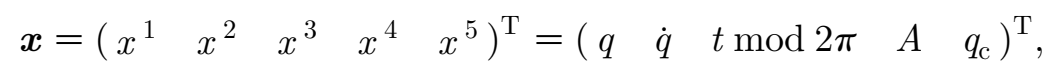

the corresponding dynamical system is given by

$$
\begin{aligned}
\frac{\mathrm{d} \boldsymbol{x}}{\mathrm{d} t}(t) & =\boldsymbol{f}(\boldsymbol{x}) \\
& =\left(\begin{array}{c}
x^{2} \\
-\frac{1}{\omega^{2}} x^{1}-\frac{2 \gamma}{\omega} x^{2}+x^{4}\left[\left(\frac{1}{\omega^{2}}-1\right) \cos x^{3}-\frac{2 \gamma}{\omega} \sin x^{3}\right] \\
1 \\
0 \\
0
\end{array}\right),
\end{aligned}
$$

as long as $h_{\mathcal{D}}(\boldsymbol{x}) \stackrel{\text { def }}{=} x^{1}-x^{5} \geq 0$ and the discontinuous change in velocity at impact is given by the jump map

$$
\boldsymbol{g}_{\mathcal{D}}(\boldsymbol{x})=\left(\begin{array}{c}
x^{1} \\
-x^{2} \\
x^{3} \\
x^{4} \\
x^{5}
\end{array}\right) .
$$

As long as $x^{4}(0) \leq-x^{5}(0)$, there exists a family of asymptotically stable periodic solutions

$$
\boldsymbol{x}(t)=\left(\begin{array}{c}
x^{4}(0) \cos t \\
-x^{4}(0) \sin t \\
t \bmod 2 \pi \\
x^{4}(0) \\
x^{5}(0)
\end{array}\right) .
$$

Indeed, when $x^{4}(0)=-x^{5}(0)=-x_{*}^{5}>0$, a grazing periodic trajectory is obtained that achieves grazing contact with the discontinuity surface $\mathcal{D}$ given by the zero-level surface of the event function $h_{\mathcal{D}}(\boldsymbol{x})$ at the point $\boldsymbol{x}_{*}=\left(\begin{array}{llllll}x_{*}^{5} & 0 & \pi & -x_{*}^{5} & x_{*}^{5}\end{array}\right)^{\mathrm{T}}$, since

$$
h_{\mathcal{D}, \boldsymbol{x}}\left(\boldsymbol{x}_{*}\right) \cdot \boldsymbol{f}\left(\boldsymbol{x}_{*}\right)=0 .
$$

In the following we are particularly concerned with bifurcations associated with changes in $x^{4}(0)$ and $x^{5}(0)$ away from $\bar{\mp} x_{*}^{5}$, respectively.

To visualize the results of the numerical and theoretical study, introduce a Poincaré section $\mathcal{P}$ corresponding to the zero-level surface of the event function $h_{\mathcal{P}}(\boldsymbol{x})=h_{\mathcal{D}, \boldsymbol{x}}(\boldsymbol{x}) \cdot \boldsymbol{f}(\boldsymbol{x})=x^{2}$, for $x^{2}$ increasing. For notational convenience, 


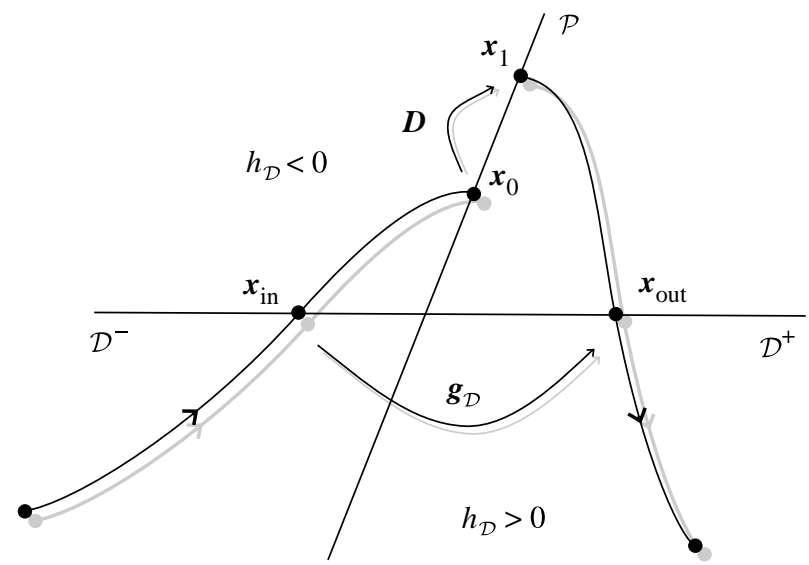

Figure 1. Schematic illustrating the correction to the smooth flow in the presence of impacts introduced through the concept of the discontinuity mapping $\boldsymbol{D}$.

consider the following definitions (cf. figure 1):

$$
\begin{gathered}
\mathcal{D}^{+}=\left\{\boldsymbol{x} \in \mathcal{D} \mid h_{\mathcal{P}}(\boldsymbol{x})>0\right\}, \\
\mathcal{D}^{0}=\left\{\boldsymbol{x} \in \mathcal{D} \mid h_{\mathcal{P}}(\boldsymbol{x})=0\right\}, \\
\mathcal{D}^{-}=\left\{\boldsymbol{x} \in \mathcal{D} \mid h_{\mathcal{P}}(\boldsymbol{x})<0\right\} .
\end{gathered}
$$

(Note that $\mathcal{P} \cap \mathcal{D}=\mathcal{D}^{0}$.) In the absence of impacts, points on $\mathcal{P}$ correspond to local minima in the value of $x^{1}$ along system trajectories (since $\dot{x}^{1}=x^{2}$ ). Since $\boldsymbol{g}_{\mathcal{D}}$ maps $\mathcal{D}^{-}$to $\mathcal{D}^{+}$, trajectories that reach $\mathcal{D}^{-}$experience an instantaneous jump across $\mathcal{P}$ to $\mathcal{D}^{+}$without intersecting $\mathcal{P}$ (as the incoming velocity $x^{2}<0$ is changed to an outgoing velocity $-x^{2}>0$ ). We represent such crossings by the virtual point of intersection with $\mathcal{P}$ of the corresponding forward trajectory segment in the absence of the jump in velocity.

\section{(b) Near-grazing dynamics}

We wish to associate a Poincaré mapping $\boldsymbol{P}$ with the Poincaré section $\mathcal{P}$ introduced above. Ignore, for a moment, the jump map associated with the discontinuity $\mathcal{D}$ and assume that the dynamics are governed entirely by the vector field $\boldsymbol{f}$. Suppose that the forward trajectory based at a point $\boldsymbol{x}_{\mathrm{ref}} \in \mathcal{P}$ intersects $\mathcal{P}$ transversally after some time $t_{\text {ref }}$, i.e. that

$$
h_{\mathcal{P}}\left(\boldsymbol{\Phi}\left(\boldsymbol{x}_{\mathrm{ref}}, t_{\mathrm{ref}}\right)\right)=0
$$

and

$$
h_{\mathcal{P}, \boldsymbol{x}}\left(\boldsymbol{\Phi}\left(\boldsymbol{x}_{\mathrm{ref}}, t_{\mathrm{ref}}\right)\right) \cdot \boldsymbol{f}\left(\boldsymbol{\Phi}\left(\boldsymbol{x}_{\mathrm{ref}}, t_{\mathrm{ref}}\right)\right)=f^{2}\left(\boldsymbol{\Phi}\left(\boldsymbol{x}_{\mathrm{ref}}, t_{\mathrm{ref}}\right)\right)>0,
$$

where $\boldsymbol{\Phi}$ is the smooth flow corresponding to the vector field $f$. Here, the latter condition corresponds to the requirement that $x^{2}$ be increasing, i.e. that the acceleration of the oscillating mass be positive.

Now introduce the function

$$
F(\boldsymbol{x}, t)=h_{\mathcal{P}}(\boldsymbol{\Phi}(\boldsymbol{x}, t))
$$


It follows that

$$
F\left(\boldsymbol{x}_{\mathrm{ref}}, t_{\mathrm{ref}}\right)=0
$$

and

$$
F_{t}\left(\boldsymbol{x}_{\text {ref }}, t_{\text {ref }}\right)>0 .
$$

The implicit function theorem implies that there exists a unique smooth function $\tau(\boldsymbol{x})$ defined on a neighbourhood of $\boldsymbol{x}_{\text {ref }}$, such that

$$
\tau\left(\boldsymbol{x}_{\mathrm{ref}}\right)=t_{\mathrm{ref}}
$$

and

$$
F(\boldsymbol{x}, \tau(\boldsymbol{x})) \equiv 0,
$$

i.e. $\tau(\boldsymbol{x})$ is the time of flight from $\boldsymbol{x}$ back to $\mathcal{P}$. A smooth Poincare mapping $\boldsymbol{P}_{\text {smooth }}$ can now be defined on a neighbourhood of $\boldsymbol{x}_{\text {ref }}$ by the expression

$$
\boldsymbol{P}_{\text {smooth }}(\boldsymbol{x})=\boldsymbol{\Phi}(\boldsymbol{x}, \tau(\boldsymbol{x})) .
$$

If we reintroduce the non-trivial jump map $\boldsymbol{g}_{\mathcal{D}}$ associated with $\mathcal{D}$, the above expression is still valid as long as $h_{\mathcal{D}}(\boldsymbol{x}) \geq 0$. If, instead, $h_{\mathcal{D}}(\boldsymbol{x})<0$, we recognize that the point $\boldsymbol{x}$ corresponds to a virtual point of intersection that cannot actually be reached by the piecewise smooth dynamical system. It would, nevertheless, be convenient if we could again define the Poincaré mapping $\boldsymbol{P}$ by the above formula, but possibly including an initial correction to account for the virtual nature of the initial point $\boldsymbol{x}$. To this end, consider the Poincaré mapping $\boldsymbol{P}$ defined by

$$
\boldsymbol{P}(\boldsymbol{x})=\boldsymbol{P}_{\text {smooth }}(\boldsymbol{D}(\boldsymbol{x})),
$$

where the discontinuity mapping $\boldsymbol{D}$ maps $\boldsymbol{x}$ to some point on $\mathcal{P}$ in such a way that the subsequent dynamics respect those of the corresponding actual trajectory.

To arrive at an expression for $\boldsymbol{D}$ consider the trajectory segments shown in figure 1 (cf. Nordmark 1991; Dankowicz \& Nordmark 1999; di Bernardo et al. 2000; Dankowicz \& Zhao 2005). Here, an incoming trajectory governed by the vector field $\boldsymbol{f}$ reaches the discontinuity surface $\mathcal{D}$ at a point $\boldsymbol{x}_{\mathrm{in}} \in \mathcal{D}^{-}$, experiences a jump to a point $\boldsymbol{x}_{\text {out }}=\boldsymbol{g}_{\mathcal{D}}\left(\boldsymbol{x}_{\text {in }}\right) \in \mathcal{D}^{+}$, and then continues to flow under the vector field $f$. The trajectory segments on the far side of the discontinuity surface $\mathcal{D}$ correspond to a flow governed by the vector field $\boldsymbol{f}$ from $\boldsymbol{x}_{\text {in }}$ forward in time until reaching $\mathcal{P}$ at a point $\boldsymbol{x}_{0}$, and from $\boldsymbol{x}_{\text {out }}$ backward in time until reaching $\mathcal{P}$ at a point $\boldsymbol{x}_{1}$. The sought correction to the smooth flow given by $\boldsymbol{\Phi}$ is then obtained by mapping $\boldsymbol{x}_{0}$ to $\boldsymbol{x}_{1}$, as this correctly accounts for the effects of the jump map.

Thus, given an initial point $\boldsymbol{x} \approx \boldsymbol{x}_{*}$ on $\mathcal{P}$, such that $h_{\mathcal{D}}(\boldsymbol{x})<0$, we define $\boldsymbol{D}$ as the composition of the following steps:

(i) flow for a time $t_{1}<0$ with the vector field $\boldsymbol{f}$ until reaching $\mathcal{D}^{-}$;

(ii) apply the jump map $\boldsymbol{g}_{\mathcal{D}}$;

(iii) flow for a time $t_{2}<0$ with the vector field $\boldsymbol{f}$ until reaching $\mathcal{P}$.

To arrive at a functional expression for $\boldsymbol{D}$, we seek to express the flow times in terms of the corresponding initial conditions in state space and the corresponding parameter values. 
Suppose, in particular, that there exists a point $\boldsymbol{x}_{*}$, such that

$$
\begin{aligned}
& h_{\mathcal{D}}\left(\boldsymbol{x}_{*}\right)=0, \\
& h_{\mathcal{P}}\left(\boldsymbol{x}_{*}\right)=0,
\end{aligned}
$$

and

$$
a^{* \text { def }}=h_{\mathcal{P}, \boldsymbol{x}}\left(\boldsymbol{x}_{*}\right) \cdot \boldsymbol{f}\left(\boldsymbol{x}_{*}\right)=f^{2}\left(\boldsymbol{x}_{*}\right)=-x_{*}^{5}>0 .
$$

Clearly, $\boldsymbol{x}_{*} \in \mathcal{P}$ is a point of simple grazing contact with $\mathcal{D}$ corresponding to a local minimum in $h_{\mathcal{D}}$ along a trajectory segment of the vector field $\boldsymbol{f}$ based at $\boldsymbol{x}_{*}$.

Step 1. Suppose that $h_{\mathcal{D}}(\boldsymbol{x})<0$ and consider the function

$$
E^{(1)}(\boldsymbol{x}, y, t)=t \sqrt{\frac{h_{\mathcal{D}}(\boldsymbol{\Phi}(\boldsymbol{x},-t))-h_{\mathcal{D}}(\boldsymbol{x})+t h_{\mathcal{P}}(\boldsymbol{x})}{t^{2}}}-y .
$$

Then, for $\boldsymbol{x} \in \mathcal{P}$, i.e. for $h_{\mathcal{P}}(\boldsymbol{x})=0, E^{(1)}\left(\boldsymbol{x}, \sqrt{-h_{\mathcal{D}}(\boldsymbol{x})}, t\right)=0$ (and the assumption that $t>0)$ implies that $h_{\mathcal{D}}(\boldsymbol{\Phi}(\boldsymbol{x},-t))=h_{\mathcal{D}}(\boldsymbol{x})+y^{2}=0$, i.e. $\boldsymbol{\Phi}(\boldsymbol{x},-t) \in \mathcal{D}$. Since

$$
\frac{h_{\mathcal{D}}(\boldsymbol{\Phi}(\boldsymbol{x},-t))-h_{\mathcal{D}}(\boldsymbol{x})+t h_{\mathcal{P}}(\boldsymbol{x})}{t^{2}}
$$

is bounded in $t$ for $t \approx 0$ (as seen by Taylor expanding the numerator in $t$ ), it follows that

$$
E^{(1)}\left(\boldsymbol{x}_{*}, 0,0\right)=0
$$

and

$$
E_{t}^{(1)}\left(\boldsymbol{x}_{*}, 0,0\right)=\sqrt{\frac{a^{*}}{2}} .
$$

The implicit function theorem now implies the existence of a unique smooth function $\boldsymbol{\tau}^{(1)}(\boldsymbol{x}, y)$ on an open neighbourhood of $\left(\boldsymbol{x}_{*}, 0\right)$, such that

$$
\tau^{(1)}\left(\boldsymbol{x}_{*}, 0\right)=0
$$

and

$$
E^{(1)}\left(\boldsymbol{x}, y, \boldsymbol{\tau}^{(1)}(\boldsymbol{x}, y)\right) \equiv 0 .
$$

It follows from the definition of $E^{(1)}$ that $t_{1}=-\tau^{(1)}\left(\boldsymbol{x}, \sqrt{-h_{\mathcal{D}}(\boldsymbol{x})}\right)$.

As the smooth function $\tau^{(1)}(\boldsymbol{x}, y)$ is implicitly defined by equation (2.29), we can compute arbitrary partial derivatives of $\tau^{(1)}$ at $\left(\boldsymbol{x}_{*}, 0\right)$ using implicit differentiation and demanding that all partial derivatives of the left-hand side of (2.29) must vanish at $\left(\boldsymbol{x}_{*}, 0,0\right)$. Now let

$$
\boldsymbol{D}_{1}(\boldsymbol{x}, y)=\boldsymbol{\Phi}\left(\boldsymbol{x},-\boldsymbol{\tau}^{(1)}(\boldsymbol{x}, y)\right) .
$$

Since $\boldsymbol{\Phi}$ is smooth, we can compute arbitrary partial derivatives of $\boldsymbol{D}_{1}$ at $\left(\boldsymbol{x}_{*}, 0\right)$. Step 1 is completed by expanding $\boldsymbol{D}_{1}(\boldsymbol{x}, y)$ to desired order in the deviation from $\left(\boldsymbol{x}_{*}, 0\right)$ and substituting $y=\sqrt{-h_{\mathcal{D}}(\boldsymbol{x})}$.

Step 2. Since $\boldsymbol{g}_{\mathcal{D}}$ is smooth, it can be expanded to desired order in the deviation from $\boldsymbol{x}_{*}$, after which the result of step 1 may be substituted for $\boldsymbol{x}$ thus completing step 2 . 
Step 3. Finally, consider the function

$$
E^{(2)}(\boldsymbol{x}, t)=h_{\mathcal{P}}(\boldsymbol{\Phi}(\boldsymbol{x},-t)) .
$$

Then,

$$
E^{(2)}\left(\boldsymbol{x}_{*}, 0\right)=h_{\mathcal{P}}\left(\boldsymbol{x}_{*}\right)=0
$$

and

$$
E_{t}^{(2)}\left(\boldsymbol{x}_{*}, 0\right)=-a^{*}<0 .
$$

The implicit function theorem again implies the existence of a unique smooth function $\tau^{(2)}(\boldsymbol{x})$ on an open neighbourhood of $\boldsymbol{x}_{*}$, such that

$$
\boldsymbol{\tau}^{(2)}\left(\boldsymbol{x}_{*}\right)=0
$$

and

$$
E^{(2)}\left(\boldsymbol{x}, \boldsymbol{\tau}^{(2)}(\boldsymbol{x})\right) \equiv 0 .
$$

It follows from the definition of $E^{(2)}$ that $t_{3}=-\tau^{(2)}(\boldsymbol{x})$.

As the smooth function $\tau^{(2)}(\boldsymbol{x})$ is implicitly defined by equation (2.35), we can compute arbitrary partial derivatives of $\boldsymbol{\tau}^{(2)}$ at $\boldsymbol{x}_{*}$ using implicit differentiation and demanding that all partial derivatives of the left-hand side of (2.35) must vanish at $\boldsymbol{x}_{*}$. Now let

$$
\boldsymbol{D}_{2}(\boldsymbol{x})=\boldsymbol{\Phi}\left(\boldsymbol{x},-\tau^{(2)}(\boldsymbol{x})\right) .
$$

Since $\boldsymbol{\Phi}$ is smooth, we can compute arbitrary partial derivatives of $\boldsymbol{D}_{2}$ at $\boldsymbol{x}_{*}$. Step 3 is completed by expanding $\boldsymbol{D}_{2}(\boldsymbol{x})$ to desired order in the deviation from $\boldsymbol{x}_{*}$ and substituting the result of step 2 for $\boldsymbol{x}$.

It follows that

$$
\boldsymbol{D}(\boldsymbol{x})= \begin{cases}\boldsymbol{x} & \text { when } h_{\mathcal{D}}(\boldsymbol{x}) \geq 0, \\ \boldsymbol{D}_{2}\left(\boldsymbol{g}_{\mathcal{D}}\left(\boldsymbol{D}_{1}\left(\boldsymbol{x}, \sqrt{-h_{\mathcal{D}}(\boldsymbol{x})}\right)\right)\right) & \text { when } h_{\mathcal{D}}(\boldsymbol{x})<0 .\end{cases}
$$

Clearly, the presence of the square-root term renders the resulting discontinuity map non-differentiable at a point $\boldsymbol{x}$, where $h_{\mathcal{D}}(\boldsymbol{x})=0$, i.e. a point of grazing contact.

The lowest-order-in- $\boldsymbol{x}-\boldsymbol{x}_{*}$, non-trivial term in the expansion of $\boldsymbol{D}$ for $\boldsymbol{x} \approx \boldsymbol{x}_{*}$ is a term of the form

$$
\beta \sqrt{-h_{\mathcal{D}, \boldsymbol{x}}\left(\boldsymbol{x}_{*}\right) \cdot\left(\boldsymbol{x}-\boldsymbol{x}_{*}\right)},
$$

where

$$
\begin{aligned}
\beta & =\left(\boldsymbol{f}\left(\boldsymbol{x}_{*}\right) \frac{h_{\mathcal{P}, \boldsymbol{x}}\left(\boldsymbol{x}_{*}\right) \cdot \boldsymbol{g}_{\mathcal{D}, x}\left(\boldsymbol{x}_{*}\right) \cdot \boldsymbol{f}\left(\boldsymbol{x}_{*}\right)}{a^{*}}-\boldsymbol{g}_{\mathcal{D}, \boldsymbol{x}}\left(\boldsymbol{x}_{*}\right) \cdot \boldsymbol{f}\left(\boldsymbol{x}_{*}\right)\right) \sqrt{\frac{2}{a^{*}}} \\
& =-2 \sqrt{\frac{2}{a^{*}}}\left(\begin{array}{lllll}
0 & 0 & 1 & 0 & 0
\end{array}\right)^{\mathrm{T}} .
\end{aligned}
$$

For small deviations from $\boldsymbol{x}_{*}$ in the direction of negative values of $h_{\mathcal{D}}$, this term results in a large stretching in a direction relative to $\boldsymbol{x}_{*}$ given by the image of the vector $\beta$ under the Jacobian $\boldsymbol{P}_{\text {smooth, } x}\left(\boldsymbol{x}_{*}\right)$ of the smooth Poincaré map. Consider 
the sequence $\xi=\left\{\xi_{n}\right\}_{n=1}^{\infty}$ defined by

$$
\boldsymbol{\xi}_{n}=h_{\mathcal{D}, \boldsymbol{x}}\left(\boldsymbol{x}_{*}\right) \cdot \underbrace{\boldsymbol{P}_{\text {smooth }, \boldsymbol{x}}\left(\boldsymbol{x}_{*}\right) \cdots \boldsymbol{P}_{\text {smooth }, \boldsymbol{x}}\left(\boldsymbol{x}_{*}\right)}_{n \text { times }} \cdot \beta \text {. }
$$

Then, as argued by Fredriksson \& Nordmark (1997), a necessary condition for the persistence of a local attractor near the grazing trajectory when $x^{4}(0) \gtrsim-x^{5}(0)$ is that $\xi$ is a non-negative sequence (but see comments in the next section). Indeed, such a local attractor can be shown to lie within a region whose size grows as the square root of the absolute value of the deviation of $x^{4}$ from $x_{*}^{4}$. Here, we refer to such a scenario as a continuous grazing bifurcation. In contrast, if $\xi_{n}<0$ for some integer $n$, no local attractor will persist. Instead, such a discontinuous grazing bifurcation is associated with a sudden jump to an impacting system attractor that persists also for $x^{4}(0) \lesssim-x^{5}(0)$.

For the present example, it is straightforward to show that

$$
\xi_{1}=\frac{x_{*}^{5} \omega\left(l_{1}^{2}-l_{2}^{2}\right)}{\sqrt{\gamma^{2}-1}} \sqrt{\frac{2}{a^{*}}}
$$

where

$$
l_{1}=\exp \left[-\pi\left(\gamma+\sqrt{\gamma^{2}-1}\right) / \omega\right], \quad l_{2}=\exp \left[-\pi\left(\gamma-\sqrt{\gamma^{2}-1}\right) / \omega\right] .
$$

It follows that a necessary condition for the persistence of a local near-grazing attractor is that $\gamma>1$.

\section{(c) Control strategy}

We now seek to introduce a feedback control to render $\xi_{n}>0$ for all $n$ by making discrete changes to the value of $x^{5}$, when the trajectory intersects the zero-level surface $\mathcal{C}$ of the event function $h_{\mathcal{C}}(\boldsymbol{x})=x^{2}$ for $x^{2}$ decreasing. Denote by $\boldsymbol{x}_{* *}=\left(\begin{array}{lllll}-x_{*}^{5} & 0 & 0 & -x_{*}^{5} & x_{*}^{5}\end{array}\right)^{\mathrm{T}}$ the intersection of the grazing periodic trajectory with $\mathcal{C}$. Now, let the proposed changes be governed by the jump map

$$
\boldsymbol{g}_{\mathcal{C}}(\boldsymbol{x})=\left(\begin{array}{c}
x^{1} \\
x^{2} \\
x^{3} \\
x^{4} \\
x_{* *}^{5}+c_{1}\left(x^{1}-x_{* *}^{1}\right)+c_{3}\left(x^{3}-x_{* *}^{3}\right)+c_{5}\left(x^{5}-x_{* *}^{5}\right)
\end{array}\right) .
$$

It follows that

$$
\boldsymbol{P}_{\text {smooth }}=\boldsymbol{P}_{\text {smooth }}^{\mathcal{C} \rightarrow \mathcal{P}} \circ \boldsymbol{g}^{\text {control }} \circ \boldsymbol{P}_{\text {smooth }}^{\mathcal{P} \rightarrow \mathcal{C}},
$$

where $\boldsymbol{P}_{\text {smooth }}^{\mathcal{P} \rightarrow \mathcal{C}}$ and $\boldsymbol{P}_{\text {smooth }}^{\mathcal{C} \rightarrow \mathcal{P}}$ correspond to the Poincaré maps from $\mathcal{P}$ to $\mathcal{C}$ and from $\mathcal{C}$ to $\mathcal{P}$, respectively. The Jacobian of $\boldsymbol{P}_{\text {smooth }}$ can then be written as a product of the Jacobians of $\boldsymbol{P}_{\text {smooth }}^{\mathcal{P} \rightarrow \mathcal{C}}, \boldsymbol{g}^{\text {control }}$ and $\boldsymbol{P}_{\text {smooth }}^{\mathcal{C} \rightarrow \mathcal{P}}$ evaluated at the appropriate points 
along the grazing trajectory. Since

$$
\boldsymbol{P}_{\mathrm{smooth}, \boldsymbol{x}}^{\mathcal{C} \rightarrow \mathcal{P}}, \boldsymbol{P}_{\mathrm{smooth}, \boldsymbol{x}}^{\mathcal{P} \rightarrow \mathcal{C}}=\left(\begin{array}{ccccc}
* & * & * & * & 0 \\
0 & 0 & 0 & 0 & 0 \\
* & * & * & * & 0 \\
0 & 0 & 0 & 1 & 0 \\
0 & 0 & 0 & 0 & 1
\end{array}\right)
$$

it follows that

$$
\boldsymbol{P}_{\text {smooth }, x}^{\mathcal{C} \rightarrow \mathcal{P}} \cdot \boldsymbol{g}_{\mathcal{C}, \boldsymbol{x}} \cdot \boldsymbol{P}_{\mathrm{smooth}, \boldsymbol{x}}^{\mathcal{P} \rightarrow \mathcal{C}}=\left(\begin{array}{ccccc}
* & * & * & * & 0 \\
0 & 0 & 0 & 0 & 0 \\
* & * & * & * & 0 \\
0 & 0 & 0 & 1 & 0 \\
* c_{1}+* c_{3} & * c_{1}+* c_{3} & * c_{1}+* c_{3} & * c_{1}+* c_{3} & c_{5}
\end{array}\right) \text {, }
$$

where ${ }^{*}$ refers to a non-trivial coefficient. Appropriate choices of values for the control parameters $c_{1}, c_{3}$ and $c_{5}$ may thus be sought that will render $\xi_{n}>0$ for all $n$. Since, for fixed values of $c_{5}$ each $\xi_{n}$ is linear in the remaining control parameters, allowable values for $c_{1}$ and $c_{3}$ lie in a region bounded by straight lines in the $c_{1}, c_{3}$ two-dimensional parameter space. Similarly, values outside such a region may be employed to render the grazing bifurcation discontinuous.

\section{(d) Comments on the continuity criterion}

As argued in Fredriksson \& Nordmark (1997), a sufficient condition for the persistence of a local attractor is that the sequence $\xi^{L}=\left\{\xi_{n}^{L}\right\}_{n=1}^{\infty}$, where

$$
\xi_{n}^{L}=h_{\mathcal{D}, \boldsymbol{x}}\left(\boldsymbol{x}_{*}\right) \cdot \underbrace{\boldsymbol{L} \cdots \boldsymbol{L}}_{n \text { times }} \cdot \beta,
$$

is non-negative for all matrices $\boldsymbol{L}$ near $\boldsymbol{P}_{\text {smooth, }}\left(\boldsymbol{x}_{*}\right)$. Equivalently, one may require that the sequence $\tilde{\xi}=\left\{\tilde{\xi}_{n}\right\}_{n=1}^{\infty}$ defined by

$$
\tilde{\xi}_{n}^{\text {def }}=h_{\mathcal{D}, \boldsymbol{x}}\left(\boldsymbol{x}_{*}\right) \cdot \boldsymbol{u}_{n},
$$

where $\boldsymbol{u}_{n}$ is a unit vector in the direction of

$$
\underbrace{\boldsymbol{P}_{\text {smooth }, \boldsymbol{x}}\left(\boldsymbol{x}_{*}\right) \cdots \cdots \boldsymbol{P}_{\text {smooth }, \boldsymbol{x}}\left(\boldsymbol{x}_{*}\right)}_{n \text { times }} \cdot \beta,
$$

is bounded from below by a positive number.

As formulated above, the quantities $\xi_{n} \rightarrow 0$ as $n \rightarrow \infty$ for fixed values of the control parameters, since the eigenvalues of $\boldsymbol{P}_{\text {smooth }, \boldsymbol{x}}$ lie within the unit circle. As a consequence, it may become numerically difficult to resolve whether $\xi_{n}>0$ for large enough values of $n$. In contrast, since $\tilde{\xi}_{n}$ must be bounded from below by a positive number for all $n$, there is no numerical difficulty in ascertaining whether 
the sufficient condition for the persistence of a local attractor is satisfied for a given set of control parameters. On the other hand, while the $\xi_{n}$ are linear in $c_{1}$ and $c_{3}$ (for a given values of $c_{5}$ ), the $\tilde{\xi}_{n}$ are nonlinear in the control parameters. In the numerical discussion below, we therefore seek values of the control parameters for which $\xi$ is a non-negative sequence, and only after the fact do we confirm that $\tilde{\xi}$ is bounded from below by a positive number.

The continuity criterion that $\tilde{\xi}$ is bounded from below by a positive number is directly related to the local stability of the grazing periodic trajectory. Indeed, as long as the continuity criterion is satisfied, a small initial perturbation from the grazing trajectory such that $x^{4}(0)=x_{*}^{4}=-x_{*}^{5}$ will result in at most one impact with the mechanical limit, and thus the stability of the grazing trajectory for $x^{4}=x_{*}^{4}$ is entirely determined by the non-impacting dynamics. As shown in Fredriksson \& Nordmark (1997), for values of $x^{4}$ away from $x_{*}^{4}$ the continuity criterion implies that the number of iterations of the composite Poincare map between successive impacts goes to infinity as $x^{4} \rightarrow x_{*}^{4}$, in essence that impacts are rare and far apart for small deviations from the grazing trajectory.

The implications of this rarity of impacts is that a control strategy that realizes the continuity criterion is particularly well suited for controlling transient dynamics that involve occasional impacts. Indeed, if it were only required that a local attractor persist as $x^{4}$ would increase beyond $x_{*}^{4}$, one might naively assume that it would suffice to choose values for $c_{1}, c_{3}$ and $c_{5}$ such that the mechanical limit would remain away from the non-impacting periodic trajectory. In the case of the linear oscillator, this would imply that

$$
x^{5}-x_{*}^{5}<-\left(x^{4}-x_{*}^{4}\right),
$$

where

$$
x^{5}-x_{*}^{5}=c_{1}\left(x^{4}-x_{*}^{4}\right)+c_{5}\left(x^{5}-x_{*}^{5}\right),
$$

i.e. that

$$
c_{1}<c_{5}-1,
$$

independently of the value of $c_{3}$. To keep the mechanical limit away from the non-impacting periodic trajectory for $x^{4}<x_{*}^{4}$, however, would result in the opposite inequality. It follows that to retain a local attractor near the grazing periodic trajectory on an open neighbourhood of $x_{*}^{4}$, values for $c_{1}, c_{3}$ and $c_{5}$ must be chosen to satisfy the continuity criterion. For example, the continuity criterion implies that the largest-in-modulus eigenvalue of $\boldsymbol{P}_{\mathrm{smooth}, \boldsymbol{x}}\left(\boldsymbol{x}_{*}\right)$ must be real and positive. But $c_{5}$ is one of the eigenvalues of $\boldsymbol{P}_{\text {smooth }, \boldsymbol{x}}\left(\boldsymbol{x}_{*}\right)$. Thus, in situations where the largest-in-modulus eigenvalues of $\boldsymbol{P}_{\text {smooth, } \boldsymbol{x}}\left(\boldsymbol{x}_{*}\right)$ in the absence of control are complex conjugates, one must choose a value for $c_{5}$ that is greater than the modulus of these eigenvalues.

\section{(e) Numerical results}

Consider the linear oscillator, such that

$$
\omega=10, \quad \gamma=0.2, \quad x_{*}^{5}=-1 .
$$

Then, in the absence of control, i.e. when $c_{1}=c_{3}=c_{5}=0$, we find $\xi_{1,2}>0$ but $\xi_{3}<0$, i.e. the grazing bifurcation is discontinuous. Figure 2 shows the impacting 

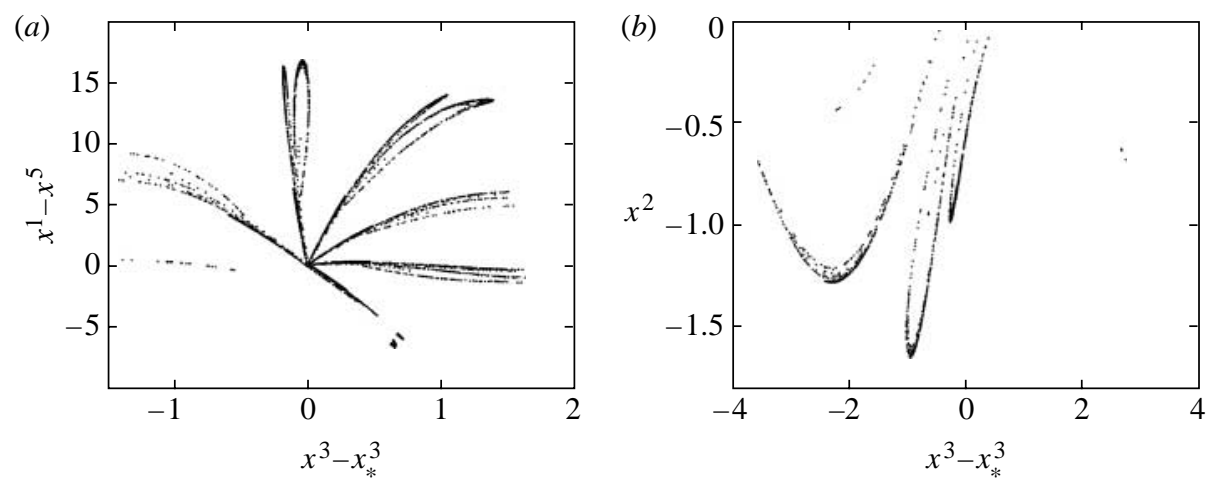

Figure 2. Chaotic impacting attractor in the near-grazing dynamics. (a) Intersection of attractor with $\mathcal{P} ;(b)$ intersection of attractor with $\mathcal{D}$.
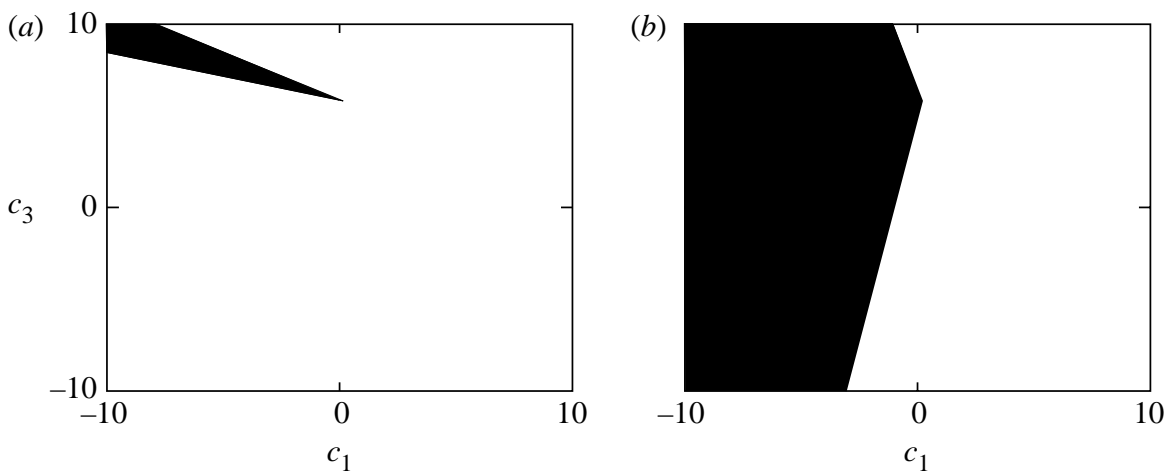

Figure 3. Shaded region represents values of $c_{1}$ and $c_{3}$, such that $\tilde{\xi}_{n}>0$ for $n \leq 90$ when $(a) c_{5}=$ 0.8819 and $(b) c_{5}=0.89$.

attractor to which an initial condition in the vicinity of the grazing trajectory converges for $x^{4}(0) \geq 1$ and which also persists for $x^{4}(0) \lesssim 1$.

In order for $\xi_{n}>0$ for all $n$, the largest-in-modulus eigenvalue of $\boldsymbol{P}_{\text {smooth, } \boldsymbol{x}}$ must be real and positive. Since, here

$$
\lambda_{1}=0, \quad \lambda_{2}=2, \quad \lambda_{3,4}=\mathrm{e}^{-\pi(1 \pm \mathrm{i} 2 \sqrt{6}) / 25}, \quad \lambda_{5}=c_{5},
$$

successful control requires that

$$
c_{5}>\mathrm{e}^{-\pi / 25} \approx 0.8819 \text {. }
$$

The shaded region in figure 3 shows a subset of the region of values for $c_{1}$ and $c_{3}$, such that $\tilde{\xi}_{n}>0$ for $n \leq 90$ when $c_{5}=0.8819$ and $c_{5}=0.89$. A bifurcation diagram showing the persistence of a local attractor in the near-grazing dynamics under variations in $x^{4}$ is shown in the upper panel of figure 4 , where $c_{1}=0, c_{3}=5.8$ and $c_{5}=0.89$. Furthermore, the lower panels of figure 4 show the local attractor with control for $x^{4}-x_{*}^{4}=10^{-5}$ as obtained from direct numerical simulations and as predicted by the composite Poincaré mapping (for the corresponding discontinuity mapping, see appendix A). 

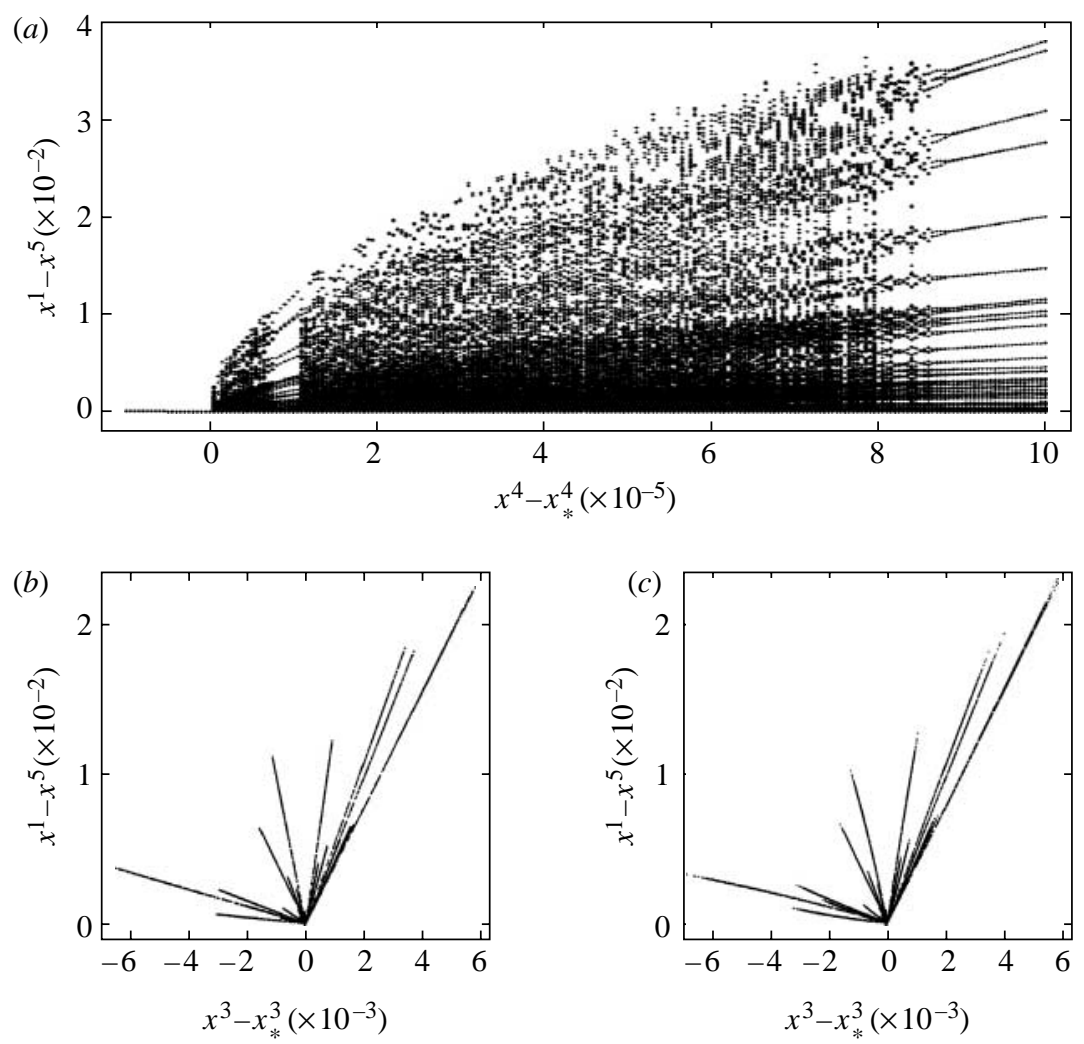

Figure 4. Near-grazing dynamics in the presence of control. (a) Bifurcation diagram under variations in $x^{4}$. Lower panels show local chaotic attractor for $x^{4}-x_{*}^{4}=10^{-5}(b)$ as obtained from direct numerical simulations and $(c)$ as predicted by the composite Poincaré mapping.

\section{A nonlinear oscillator}

Now consider the response of a nonlinear oscillator to a periodic excitation in the presence of elastic impacts of the oscillator with a mechanical limit at some critical displacement. In particular, suppose that the evolution of the oscillator is governed by the second-order differential equation

$$
\frac{\mathrm{d}^{2} q}{\mathrm{~d} t^{2}}(t)+\gamma \frac{\mathrm{d} q}{\mathrm{~d} t}(t)+q(t)=\frac{A \cos ^{2} \omega t}{(1-q(t))^{2}},
$$

for $q<q_{\mathrm{c}}<1, \omega, \gamma, A>0$, and such that if

$$
q\left(\lim _{t \rightarrow t_{*}^{-}} t\right)=q_{\mathrm{c}} \text { and } \frac{\mathrm{d} q}{\mathrm{~d} t}\left(\lim _{t \rightarrow t_{*}^{-}} t\right) \geq 0,
$$

for some time $t_{*}$, then

$$
q\left(\lim _{t \rightarrow t_{*}^{+}} t\right)=q_{\mathrm{c}} \text { and } \frac{\mathrm{d} q}{\mathrm{~d} t}\left(\lim _{t \rightarrow t_{*}^{+}} t\right)=-e \frac{\mathrm{d} q}{\mathrm{~d} t}\left(\lim _{t \rightarrow t_{*}^{-}} t\right),
$$

where $e$ represents a coefficient of restitution. In terms of the state vector

$$
\boldsymbol{x}=\left(\begin{array}{lllll}
x^{1} & x^{2} & x^{3} & x^{4} & x^{5}
\end{array}\right)^{\mathrm{T}}=\left(\begin{array}{lllll}
q & \dot{q} & \omega t \bmod \pi & A & q_{\mathrm{c}}
\end{array}\right)^{\mathrm{T}},
$$


the corresponding dynamical system is given by

$$
\frac{\mathrm{d} \boldsymbol{x}}{\mathrm{d} t}(t)=\boldsymbol{f}(\boldsymbol{x})=\left(\begin{array}{c}
x^{2} \\
-x^{1}-\gamma x^{2}+x^{4} \frac{\cos ^{2} x^{3}}{\left(1-x^{1}\right)^{2}} \\
\omega \\
0 \\
0
\end{array}\right),
$$

as long as $h_{\mathcal{D}}(\boldsymbol{x}) \stackrel{\text { def }}{=} x^{5}-x^{1} \geq 0$ and the discontinuous change in velocity at impact is given by the jump map

$$
\boldsymbol{g}_{\mathcal{D}}(\boldsymbol{x})=\left(\begin{array}{c}
x^{1} \\
-e x^{2} \\
x^{3} \\
x^{4} \\
x^{5}
\end{array}\right)
$$

Following the discussion in the case of the linear oscillator, introduce a Poincaré section $\mathcal{P}$ given by the zero-level surface of the event function $h_{\mathcal{P}}(\boldsymbol{x})=$ $h_{\mathcal{D}, x}(\boldsymbol{x}) \cdot \boldsymbol{f}(\boldsymbol{x})=-x^{2}$ for $x^{2}$ decreasing. Again, we may analyse the dynamics in the vicinity of a grazing trajectory by composing the smooth Poincaré map $\boldsymbol{P}_{\text {smooth }}$ associated with $\mathcal{P}$ in the absence of impacts with a discontinuity mapping $\boldsymbol{D}$ that (i) captures the local dynamics in the vicinity of the grazing contact including variations in time-of-flight to the discontinuity and the impact mapping; (ii) can be entirely characterized by conditions at the grazing contact; (iii) is non-smooth in the deviation from the point of grazing contact; and (iv) can be studied to arbitrary order of accuracy. Properly formulated, the discontinuity mapping thus introduces the correction to the otherwise smooth dynamics that is due to the brief interaction with the discontinuity.

Suppose that $\gamma=0.04, \omega=0.56, e=0.8$. Then, a grazing periodic trajectory is obtained for

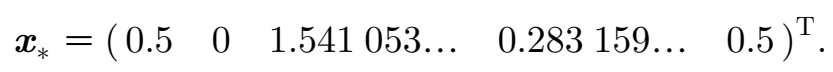

In this case, $\xi_{1}<0$ in the absence of control and the corresponding grazing bifurcation is thus discontinuous. Indeed, the left panel of figure 5 shows the impacting periodic attractor to which an initial condition in the vicinity of the grazing trajectory converges for $x^{4}(0) \geq x_{*}^{4}$ and which also persists for $x^{4}(0) \leqq x_{*}^{4}$.

Here, the largest-in-modulus eigenvalues of $\boldsymbol{P}_{\text {smooth, } \boldsymbol{x}}\left(\boldsymbol{x}_{*}\right)$ for $c_{5}=0$ are complex conjugate with modulus $\approx 0.8939$. Let $c_{5}=0.95$. Then, the right panel of figure 5 shows a subset of the region of values for $c_{1}$ and $c_{3}$, such that $\tilde{\xi}_{n}>0$ for $n \leq 90$. As an example, figure 6 shows the grazing bifurcations scenario that is obtained when $c_{1}=0$ and $c_{3}=-1.4$. Specifically, while the upper panel shows the predictions of an analytical expansion of $\boldsymbol{P}$ to linear order in the deviation $\boldsymbol{x}-\boldsymbol{x}_{*}$, the lower panel shows the results of applying the control to the original dynamical system over a larger range in the deviation $x^{4}-x_{*}^{4}$. 

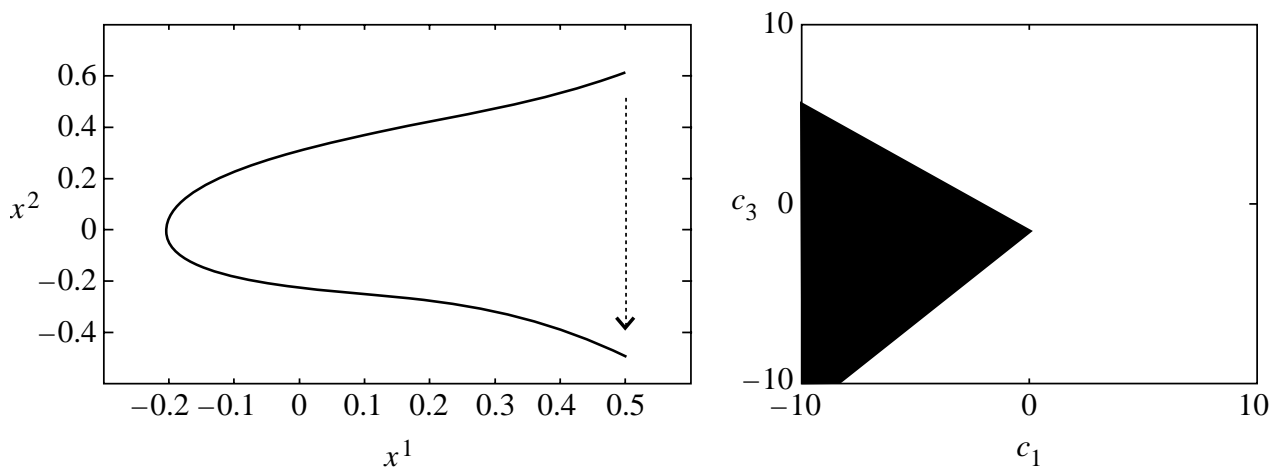

Figure 5. (a) Near-grazing dynamics in the absence of control and (b) subset of values of $c_{1}$ and $c_{3}$, such that $\tilde{\xi}_{n}>0$ for $n \leq 90$ when $c_{5}=0.95$.

\section{Conclusions}

This paper has presented a linear, discrete, closed-loop control strategy for ensuring the persistence of a local attractor in the near-grazing dynamics of an impact oscillator. Although the methodology has been presented specifically for forced, single-degree-of-freedom oscillators, there is nothing in the approach that limits it to this low dimensionality or to systems that originate in second-order differential equations. Indeed, the discontinuity-mapping approach as well as the continuity criterion formulated here are general to near-grazing dynamics in arbitrary-dimensional piecewise-smooth dynamical systems.

Of course, the higher the dimension of the underlying dynamical system, the more control parameters would enter into the expression for the feedback strategy. Again, however, for fixed values of the control parameter coupling the change in the position of the mechanical limit to the current position of the mechanical limit ( $c_{5}$ in the above analysis), the resultant expressions for the elements of the sequence $\xi$ would be linear in the remaining control parameters. One would thus, again, expect that allowable values for the remaining control parameters would lie in a region in parameter space bounded by hyperplanes. As noted above, in a situation where the largest-in-modulus eigenvalues of the Jacobian of the smooth Poincaré map in the absence of control were complex conjugate, it would be necessary to select the control parameter coupling the change in the position of the mechanical limit to the current position of the mechanical limit to be larger than the modulus of these eigenvalues (but always less than one).

The control of system dynamics in the presence of impacts using the position of the system discontinuity has previously been employed by the authors (Dankowicz \& Piiroinen 2002; Jerrelind \& Dankowicz in press). There, however, the analysis focused on the stabilization of impacting dynamics in the presence of large-impactvelocity motions, far from the near-grazing region. In that case, the local dynamics were described by a linear Poincaré map and the analysis focused on the control of the linearized system about an impacting reference trajectory. In contrast, in the situation considered here, the stabilization of a particular trajectory is practically impossible. Instead, the aim of the present work has been to change the bifurcation behaviour in the near-grazing region ensuring, not the stability of a single trajectory, but the weaker notion of stability pertaining to the persistence of a local attractor. 

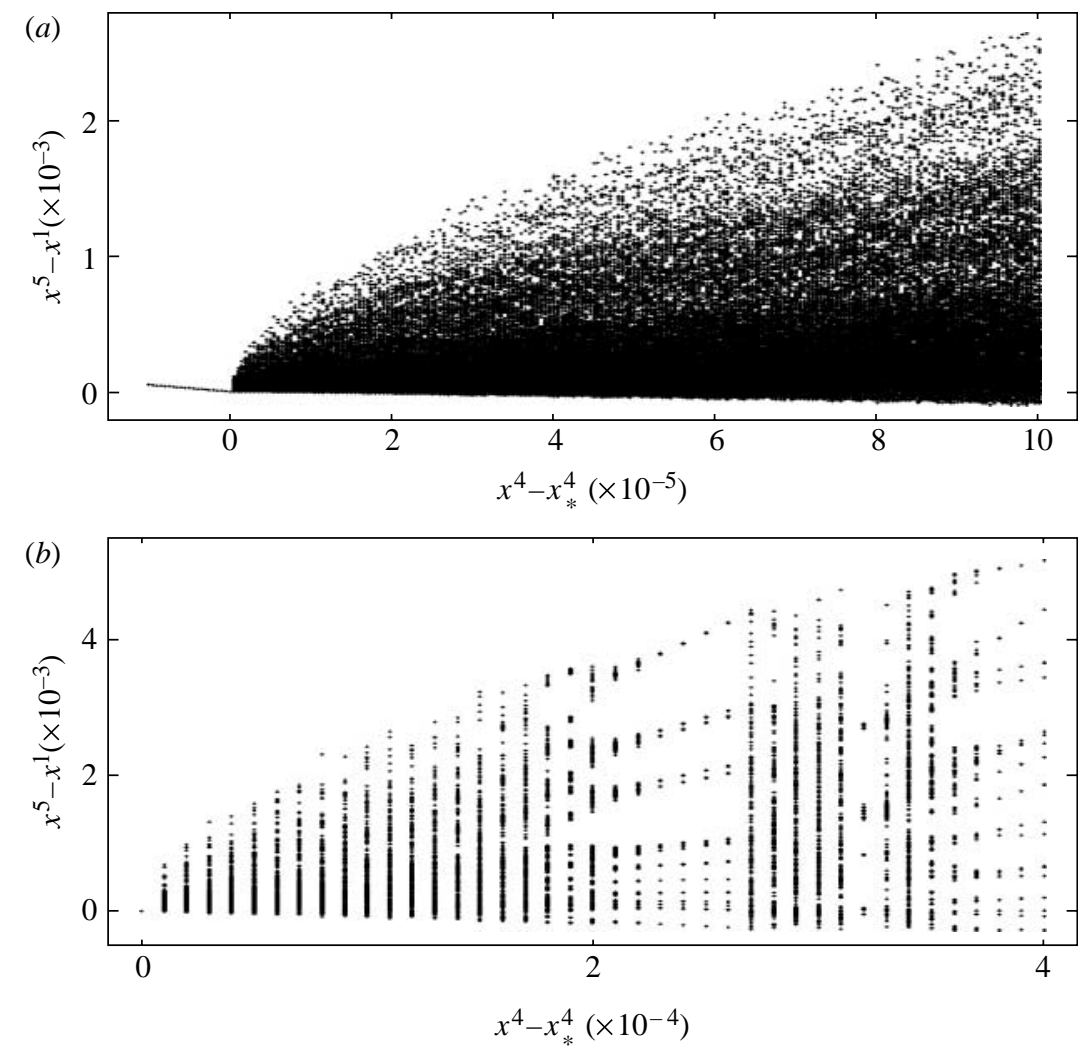

Figure 6. Near-grazing dynamics in the presence of control. Bifurcation diagrams under variations in $x^{4}(a)$ as predicted by the composite Poincaré mapping and $(b)$ as obtained from direct numerical simulations.

The proposed control strategy affords a means of suppressing the catastrophic loss of stability of low-impact-velocity motion to high-impact-velocity motion that is a distinct possibility in impact oscillators. Instead, at the expense of small, real-time and closed-loop adjustments to the position of the system discontinuity, low-impact-velocity motions may be sustained over a large interval about the point of grazing contact. The implications of this to practical mechanical and/or electrical systems will be explored in future work.

This material is based upon work supported by the National Science Foundation under grant no. 0237370 and by the Swedish Science Council, Division of Engineering Mechanics, grant numbers 2003-3699 and 2004-6342.

\section{Appendix A. Explicit expansions for the discontinuity mappings}

As suggested in the main text, implicit differentiation may be employed to obtain expressions for the coefficients in the various Taylor expansions in the construction of the discontinuity mapping $\boldsymbol{D}$. As an example of the result of such computations, the formulae below correspond to the discontinuity mappings for the linear and nonlinear oscillator, respectively, for $\boldsymbol{x} \approx \boldsymbol{x}_{*}$. Since $\boldsymbol{D}$ maps $\mathcal{P}$ to 
$\mathcal{P}$ and since $x^{4}$ and $x^{5}$ are constant under the smooth flow, we only report the non-trivial 1 and 3 components of $\boldsymbol{D}$.

Specifically, in the case of the linear oscillator with

$$
\omega=10, \quad \gamma=0.2, \quad x_{*}=\left(\begin{array}{lllll}
-1 & 0 & \pi & 1 & -1
\end{array}\right)^{\mathrm{T}},
$$

we find

$$
\begin{aligned}
D^{1}(\boldsymbol{x}) & \approx x^{1}{ }^{1}+\Delta x^{1}, \\
D^{3}(\boldsymbol{x}) & \approx x_{*}^{3}-2 \sqrt{2} \sqrt{\Delta x^{5}-\Delta x^{1}}-\frac{4}{25}\left(\Delta x^{5}-\Delta x^{1}\right)+\Delta x^{3},
\end{aligned}
$$

where $\boldsymbol{\Delta} \boldsymbol{x}=\boldsymbol{x}-\boldsymbol{x}_{*}$ and we have included terms up to linear order in the deviation $\boldsymbol{\Delta} \boldsymbol{x}$.

Similarly, in the case of the nonlinear oscillator with

$$
\begin{aligned}
& \gamma=0.04, \quad \omega=0.56, \quad e=0.8,
\end{aligned}
$$

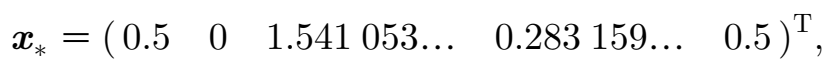

we find

$$
\begin{aligned}
& D^{1}(\boldsymbol{x}) \approx x_{*}^{1}+0.6400 \Delta x^{1}+0.3600 \Delta x^{3} \\
& D^{3}(\boldsymbol{x}) \approx x_{*}^{3}-2.0180 \sqrt{\Delta x^{1}-\Delta x^{5}}-0.2107\left(\Delta x^{1}-\Delta x^{5}\right)+\Delta x^{3} .
\end{aligned}
$$

Note that to obtain an expansion for the composite Poincaré mapping to first-order in the deviation from the point of grazing contact, it is necessary to compute expansions of the corresponding smooth Poincaré mappings to second-order. This is a straightforward application of the definition of the smooth Poincaré mapping and the solution to the first and second variational equations for the smooth flow.

\section{References}

Dankowicz, H. \& Nordmark, A. B. 1999 On the origin and bifurcations of stick-slip oscillations. Physica D 136, 280-302.

Dankowicz, H. \& Piiroinen, P. 2002 Exploiting discontinuities for stabilization of recurrent motions. Dyn. Syst. 17, 317-342.

Dankowicz, H. \& Zhao, X. 2005 Local analysis of co-dimension-one and co-dimension-two grazing bifurcations in impact microactuators. Physica D 202, 238-257.

Dankowicz, H., Piiroinen, P. \& Nordmark, A. B. 2002 Low-velocity impacts of quasiperiodic oscillations. Chaos Solitons Fractals 14, 241-255. (doi:10.1016/S0960-0779(01)00230-2.)

di Bernardo, M., Budd, C. J. \& Champneys, A. R. 2000 Normal form maps for grazing bifurcations in $n$-dimensional piecewise-smooth dynamical systems. Physica D 160, 222-254.

Fredriksson, M. H. \& Nordmark, A. B. 1997 Bifurcations caused by grazing incidence in many degrees of freedom impact oscillators. Proc. R. Soc. A 453, 1261-1276. (doi:10.1098/rspa.1997.0069.)

Jerrelind, J. \& Dankowicz, H. In press. A global control strategy for efficient control of a Braille impact hammer. J. Vibr. Acoust.

Molenaar, J., de Weger, J. G. \& Van de Water, W. 2001 Mappings of grazing impact oscillators. Nonlinearity 14, 301-321. (doi:10.1088/0951-7715/14/2/307.)

Nordmark, A. B. 1991 Non-periodic motion caused by grazing incidence in an impact oscillator. J. Sound Vibr. 145, 279-297. (doi:10.1016/0022-460X(91)90592-8.)

Nordmark, A. B. 1997 Universal limit mapping in grazing bifurcations. Phys. Rev. E 55, 266-270. (doi:10.1103/PhysRevE.55.266.) 